\title{
Extremality Properties for Gallager's Random Coding Exponent
}

\author{
Mine Alsan \\ LTHI, I\&C, EPFL \\ Lausanne, Switzerland \\ Email: mine.alsan@epfl.ch
}

\begin{abstract}
We describe certain extremality properties for Gallager's reliability function $E_{0}$ for binary input symmetric DMCs. In particular, we show that amongst such DMC's whose $E_{0}\left(\rho_{1}\right)$ has a given value for a given $\rho_{1}$, the BEC and BSC have the largest and smallest value of the derivative of $E_{0}\left(\rho_{2}\right)$ for any $\rho_{2} \geq \rho_{1}$. As the random coding exponent is obtained by tracing the map $\rho \rightarrow\left(E_{0}^{\prime}(\rho), E_{0}(\rho)-\rho E_{0}^{\prime}(\rho)\right)$ this conclusion includes as a special case the results of [1]. Furthermore, we show that amongst channels $W$ with a given value of $E_{0}(\rho)$ for a given $\rho$ the BEC and BSC are the most and least polarizing under Arıkan's polar transformations in the sense that their polar transforms $W^{+}$and $W^{-}$has the largest and smallest difference in their $E_{0}$ values.

Index Terms-Channel reliability function, random coding exponent, channel polarization.
\end{abstract}

\section{INTRODUCTION}

While the capacity of a memoryless channel $W$ gives the largest rate that may be communicated reliably across it, the reliability function $E(R, W)$ provides a finer measure on the quality of the channel: for any rate $R$ less than channel capacity, it is possible to find a sequence of codes of increasing blocklength, each of which of rate at least $R$, and whose block error probability decays exponentially to zero in the blocklength $-E(R, W)$ is the largest possible rate of this decay.

Gallager classical treatise [2] gives a lower bound to $E(R, W)$, the random coding exponent $E_{r}(R, W)$ in the form $E_{r}(R, W)=\max _{\rho \in[0,1]} E_{0}(\rho, W)-\rho R$. Remarkably, this lower bound is tight for rates above the critical rate $E_{0}^{\prime}(1, W)$. The function $E_{0}(\rho, W)$ that appears as an auxiliary function on the road to deriving $E_{r}(R, W)$ turns out to be of independent interest in its own right. In particular, $E_{0}(\rho, W) / \rho$ is the largest rate for which a sequential decoder can operate while keeping the $\rho$-th moment of the decoder's computation effort per symbol bounded.

In this paper we investigate the extremal properties of $E_{0}(\rho, W)$ for the class of binary input symmetric channels. We show that among all such channels with a given value of $E_{0}\left(\rho_{1}, W\right)$ the binary erasure channel (BEC) and the binary symmetric channel (BSC) distinguish themselves in certain ways: they have, respectively, the largest and smallest value of $E_{0}^{\prime}\left(\rho_{2}, W\right)$ for any $\rho_{2} \geq \rho_{1}$. Among the simple corollaries of this is the conclusion that of all the channels with the same capacity, the BEC and BSC have the largest and smallest value of $E_{r}(R, W)$, a result reported in [1] last year.
The BEC and BSC also exhibit themselves as being extremal for Arıkan's polarization transforms. In his award winning paper [3], Arıkan describes two synthetic channels $W^{+}$, and $W^{-}$which can be obtained from two independent copies of $W$. It is well known (proved as a corollary to extremes of information combining) that among all channels $W$ with a given symmetric capacity $I(W)$, the BEC and BSC polarize most and least in the sense of having the largest and smallest difference between $I\left(W^{+}\right)$and $I\left(W^{-}\right)$. We report a more general conclusion: amongst all channels $W$ with a given value of $E_{0}(\rho, W)$, the BEC and BSC polarize most and least in the sense of having the largest difference between $E_{0}\left(\rho, W^{+}\right)$and $E_{0}\left(\rho, W^{-}\right)$.

\section{EXTREMALity OF THE RANDOM CODING EXPONENT}

\section{A. Definitions and Properties}

Definition 1: [2] Given a discrete memoryless channel $W$ with input alphabet $\mathcal{X}$ and output alphabet $\mathcal{Y}$, fix a distribution $Q$ on its input alphabet.

Consider the function $E_{r}(R, Q, W)$ defined as

$$
E_{r}(R, Q, W)=\max _{\rho \in[0,1]}\left\{E_{0}(\rho, Q, W)-\rho R\right\}
$$

for $R \geq 0$, with

$$
E_{0}(\rho, Q, W)=-\log \sum_{y \in \mathcal{Y}}\left[\sum_{x \in \mathcal{X}} Q(x) W(y \mid x)^{\frac{1}{1+\rho}}\right]^{1+\rho} .
$$

The random coding exponent is defined as

$$
E_{r}(R, W)=\max _{Q} E_{r}(R, Q, W) .
$$

We restrict our attention to symmetric binary input channels (B-DMCs). In this case the uniform input distribution maximizes Equation (3). Accordingly, we drop the variable $Q$ from the notations. The expression in (2) becomes

$$
E_{0}(\rho, W)=-\log \sum_{y \in \mathcal{Y}}\left[\frac{1}{2} W(y \mid 0)^{\frac{1}{1+\rho}}+\frac{1}{2} W(y \mid 1)^{\frac{1}{1+\rho}}\right]^{1+\rho} .
$$

Theorem 5.6.3 in [2] summarizes the properties of $E_{0}(\rho, W)$ with respect to the variable $\rho$. For $\rho \geq 0, E_{0}(\rho, W)$ is a positive, concave increasing function in $\rho$. Moreover, the symmetric capacity $I(W)$ and the Bhattacharyya parameter $Z(W)$ of the channel can be derived from $E_{0}(\rho, W)$ by 
- $\left.\frac{\partial}{\partial \rho} E_{0}(\rho, W)\right|_{\rho=0}=\left.\frac{E_{0}(\rho, W)}{\rho}\right|_{\rho=0}=I(W)$

- $E_{0}(1, W)=\log \frac{2}{1+Z(W)}$.

The maximization in the right hand side of Equation (1) over $\rho \in[0,1]$ can be described in terms of the following parametric equations:

$$
\begin{aligned}
& R(\rho, W)=\frac{\partial}{\partial \rho} E_{0}(\rho, W) \\
& E_{r}(\rho, W)=E_{0}(\rho, W)-\rho \frac{\partial}{\partial \rho} E_{0}(\rho, W)
\end{aligned}
$$

for $R$ in the range $\left.\frac{\partial E_{0}(\rho, W)}{\partial \rho}\right|_{\rho=1} \leq R \leq\left.\frac{\partial E_{0}(\rho, W)}{\partial \rho}\right|_{\rho=0}$.

\section{B. Extremality of the Random Coding Exponent}

We show in this section that the binary erasure channel and the binary symmetric channel are extremal among all symmetric B-DMCs with respect to the random coding exponent. In particular, we show in Theorem 1 a certain extremality property holds even when the quantities appearing in the parametric form of the error exponent are allowed to be evaluated at different values of the parameter.

Theorem 1: Given a symmetric B-DMC $W$, for any fixed value of $\rho_{1} \in[0,1]$, we define a binary symmetric channel $W_{\mathrm{BSC}}$, and a binary erasure channel $W_{\mathrm{BEC}}$ through the equality

$$
E_{0}\left(\rho_{1}, W\right)=E_{0}\left(\rho_{1}, W_{\mathrm{BSC}}\right)=E_{0}\left(\rho_{1}, W_{\mathrm{BEC}}\right) .
$$

Then, for any $\rho_{2} \in\left[\rho_{1}, 1\right]$, we have

$$
R\left(\rho_{2}, W_{\mathrm{BSC}}\right) \leq R\left(\rho_{2}, W\right) \leq R\left(\rho_{2}, W_{\mathrm{BEC}}\right) .
$$

The proof of the theorem is given in Appendix VI-A.

Remark 1: The erasure probability of $W_{\mathrm{BEC}}$ and the crossover probability of $W_{\mathrm{BSC}}$ depend both on the channel $W$ and the parameter $\rho_{1}$.

For the special case when $\rho_{1}=\rho_{2}$, we recover in the next Corollary a result obtained in [4].

Corollary 1 ([4]): Given a symmetric B-DMC $W$, for any fixed value of $\rho \in[0,1]$, we define a binary symmetric channel $\tilde{W}_{\text {BSC }}$, and a binary erasure channel $\tilde{W}_{\text {BEC }}$ through the equality

$$
R(\rho, W)=R\left(\rho, \tilde{W}_{\mathrm{BEC}}\right)=R\left(\rho, \tilde{W}_{\mathrm{BSC}}\right) .
$$

Then,

$$
\begin{aligned}
& E_{0}\left(\rho, \tilde{W}_{\mathrm{BEC}}\right) \leq E_{0}(\rho, W) \leq E_{0}\left(\rho, \tilde{W}_{\mathrm{BSC}}\right) \\
& E_{r}\left(\rho, \tilde{W}_{\mathrm{BEC}}\right) \leq E_{r}(\rho, W) \leq E_{r}\left(\rho, \tilde{W}_{\mathrm{BSC}}\right) .
\end{aligned}
$$

Proof: Since $E_{r}(\rho, W)=E_{0}(\rho, W)-\rho R(\rho, W)$, it suffices to prove the first set of inequalities in view of (8). For a fixed value of $\rho$, we define another binary erasure channel $W_{\mathrm{BEC}}$ through the equality

$$
E_{0}(\rho, W)=E_{0}\left(\rho, W_{\mathrm{BEC}}\right) .
$$

By Theorem 1, we know that $R(\rho, W) \leq R\left(\rho, W_{\mathrm{BEC}}\right)$. Via (8), we thus get $R\left(\rho, \tilde{W}_{\mathrm{BEC}}\right) \leq R\left(\rho, W_{\mathrm{BEC}}\right)$. As a result,

$$
E_{0}\left(\rho, \tilde{W}_{\mathrm{BEC}}\right) \leq E_{0}\left(\rho, W_{\mathrm{BEC}}\right)=E_{0}(\rho, W) .
$$

The inequality for the binary symmetric channel can be obtained similarly.

Another particular case of Theorem 1 when $\rho_{1} \rightarrow 0$ recovers the result in [1]: among all symmetric B-DMCs of the same capacity, the binary erasure channel and the binary symmetric channel are extremal with respect to the random coding exponent. This is shown in the next corollary.

Corollary 2 (Theorem 2.3 [1]): Given a symmetric BDMC $W$ of capacity $I(W)$, we define a binary symmetric channel $W_{\mathrm{BSC}}$, and a binary erasure channel $W_{\mathrm{BEC}}$ of the same capacity through the equality

$$
I(W)=I\left(W_{\mathrm{BEC}}\right)=I\left(W_{\mathrm{BSC}}\right) .
$$

Then, the random coding error exponent of the channels satisfy

$$
E_{r}\left(\rho, W_{\mathrm{BSC}}\right) \leq E_{r}(\rho, W) \leq E_{r}\left(\rho, W_{\mathrm{BEC}}\right) .
$$

Proof: Same capacity is equivalent to

$$
\left.\frac{E_{0}\left(\rho_{1}, W\right)}{\rho_{1}}\right|_{\rho_{1}=0}=\left.\frac{E_{0}\left(\rho_{1}, W_{\mathrm{BEC}}\right)}{\rho_{1}}\right|_{\rho_{1}=0}=\left.\frac{E_{0}\left(\rho_{1}, W_{\mathrm{BSC}}\right)}{\rho_{1}}\right|_{\rho_{1}=0} .
$$

Moreover, we can reformulate Theorem 1 by replacing the equality condition imposed for the functions $E_{0}(\rho, W)$ in Equation (6), by equality among the functions $\frac{E_{0}(\rho, W)}{\rho}$. Since this latter is a continuous function of $\rho$, we deduce that the result of the theorem is still valid for $\rho_{1} \rightarrow 0$. As a consequence, for any $\rho_{2} \in[0,1]$, we have

$$
R\left(\rho_{2}, W_{\mathrm{BSC}}\right) \leq R\left(\rho_{2}, W\right) \leq R\left(\rho_{2}, W_{\mathrm{BEC}}\right) .
$$

As $\frac{\partial}{\partial \rho} E_{0}(\rho, W)=R(\rho, W)$, this last inequality implies that

$$
E_{0}\left(\rho, W_{\mathrm{BSC}}\right) \leq E_{0}(\rho, W) \leq E_{0}\left(\rho, W_{\mathrm{BEC}}\right)
$$

which, in turn, implies the inequality for the random coding exponent.

\section{EXTREMALITY FOR THE POLARIZATION TRANSFORMATIONS}

In this section, we study the behavior of $E_{0}(\rho, W)$ from the aspect of channel polarization. In Theorem 2, we show that the binary erasure channel and the binary symmetric channel are also extremal in the evolution of $E_{0}(\rho, W)$ under the polarization transformations.

\section{A. Basic Polarization Transformations}

In [3], a low complexity code construction that achieves the capacity of symmetric B-DMCs is given based on the recursive application of two basic channel transformations. These transforms synthesize two new channels $W^{-}: \mathcal{X} \rightarrow \mathcal{Y}^{2}$ and $W^{+}: \mathcal{X} \rightarrow \mathcal{Y}^{2} \times \mathcal{X}$ by combining two copies of the channel $W$. The channels are defined by

$$
\begin{aligned}
& W^{-}\left(y_{1} y_{2} \mid u_{1}\right)=\sum_{u_{2} \in \mathcal{X}} \frac{1}{2} W\left(y_{1} \mid u_{1} \oplus u_{2}\right) W\left(y_{2} \mid u_{2}\right) \\
& W^{+}\left(y_{1} y_{2} u_{1} \mid u_{2}\right)=\frac{1}{2} W\left(y_{1} \mid u_{1} \oplus u_{2}\right) W\left(y_{2} \mid u_{2}\right) .
\end{aligned}
$$


Arıkan shows that the recursive application of these transforms polarize any $W$ in the sense that almost all synthesized channels are either almost perfect or almost very noisy. Intuitively, one expects that $E_{0}(\rho) / \rho$ similarly polarizes. The next proposition provides a simple proof for this argument.

Proposition 1: After the recursive application of the polarization transformations, the $E_{0}(\rho) / \rho$ parameters of the synthesized channels polarize to the extremal values $\{0,1\}$ when a long sequence of steps is applied to the channel.

Proof: In [5], it is shown that $E_{0}(\rho, W) / \rho$ is a decreasing function in $\rho$. Therefore, we can squeeze the function between

$$
-\log \left(\frac{1+Z(W)}{2}\right) \leq \frac{E_{0}(\rho, W)}{\rho} \leq I(W) .
$$

The proof follows based on the fact that the quantities $I(W)$, and $Z(W)$ both polarize, i.e., $I(W) \rightarrow 0 \Longleftrightarrow Z(W) \rightarrow 1$, and $I(W) \rightarrow 1 \Longleftrightarrow Z(W) \rightarrow 0$ [3].

Remark 2: Based on numerical experiments, we conjecture the channels $W, W^{-}$, and $W^{+}$satisfy the next relationship:

$$
E_{0}\left(\rho, W^{-}\right)+E_{0}\left(\rho, W^{+}\right) \geq 2 E_{0}(\rho, W) .
$$

Remark 3: [6] For a symmetric $W$ the channels $W^{-}, W$, and $W^{+}$are ordered by degradation. Consequently,

$$
E_{0}\left(\rho, W^{-}\right) \leq E_{0}(\rho, W) \leq E_{0}\left(\rho, W^{+}\right) .
$$

\section{B. Extremality of the Basic Channel Transformations}

Theorem 2: Given a symmetric B-DMC $W$, for any fixed value of $\rho \in[0,1]$, we define (as in Theorem 1) a binary symmetric channel $W_{\mathrm{BSC}}$, and a binary erasure channel $W_{\mathrm{BEC}}$ through the equality

$$
E_{0}(\rho, W)=E_{0}\left(\rho, W_{\mathrm{BEC}}\right)=E_{0}\left(\rho, W_{\mathrm{BSC}}\right) .
$$

Then

$$
\begin{aligned}
& E_{0}\left(\rho, W_{\mathrm{BEC}}^{-}\right) \leq E_{0}\left(\rho, W^{-}\right) \leq E_{0}\left(\rho, W_{\mathrm{BSC}}^{-}\right) \\
& E_{0}\left(\rho, W_{\mathrm{BSC}}^{+}\right) \leq E_{0}\left(\rho, W^{+}\right) \leq E_{0}\left(\rho, W_{\mathrm{BEC}}^{+}\right) .
\end{aligned}
$$

The proof is given in Appendix VI-B.

In Theorem 2, we have shown that among all B-DMC's $W$ of fixed $E_{0}(\rho, W)$, the binary erasure channel $W^{-}$transformation results in a lower bound to any $E_{0}\left(\rho, W^{-}\right)$and the binary symmetric channel's one in an upper bound to any $E_{0}\left(\rho, W^{-}\right)$. For the $W^{+}$transformation, a similar extremality property holds except the difference that the binary erasure channel $W^{+}$transformation provides an upper bound and the binary symmetric channel's one a lower bound to any $E_{0}\left(\rho, W^{+}\right)$. This shows that the binary erasure and binary symmetric channels appear on reversed sides of the inequalities for $E_{0}\left(\rho, W^{-}\right)$and $E_{0}\left(\rho, W^{+}\right)$.

Corollary 3: Under the same assumptions as Theorem 2

$$
\begin{array}{r}
E_{0}\left(\rho, W_{\mathrm{BSC}}^{+}\right)-E_{0}\left(\rho, W_{\mathrm{BSC}}^{-}\right) \leq E_{0}\left(\rho, W^{+}\right)-E_{0}\left(\rho, W^{-}\right) \\
\leq E_{0}\left(\rho, W_{\mathrm{BEC}}^{+}\right)-E_{0}\left(\rho, W_{\mathrm{BEC}}^{-}\right) .
\end{array}
$$

Remark 4: Dividing all sides of the inequality above by $\rho$ and taking the limit as $\rho \rightarrow 0$, we see that among channels of a given symmetric capacity the BEC and BSC are extremal with respect to the polarization transformations, in the sense that

$$
\begin{aligned}
I\left(W_{\mathrm{BSC}}^{+}\right)-I\left(W_{\mathrm{BSC}}^{-}\right) \leq I\left(W^{+}\right) & -I\left(W^{-}\right) \\
& \leq I\left(W_{\mathrm{BEC}}^{+}\right)-I\left(W_{\mathrm{BEC}}^{-}\right) .
\end{aligned}
$$

These inequalities can also be obtained by the results on the extremes of information combining [7], together with the fact that symmetric capacity is preserved under the polarization transformations [3]. Note also that, when combined with the preservation property, only one of the Equations (13) and (14) would be sufficient to get the result.

\section{CONCLUSions}

We have described some extremality properties for binary input channels when the information measure is Gallager's $E_{0}$. These properties yield in straightforward fashion already known extremality results.

The extremality of BEC and BSC for polar transforms can be interpreted in the context of information combining. Theorem 2 shows that even if we change the measure of information from the customary mutual information to $E_{0}$ the channels BEC and BSC still remain extremal.

The assumed symmetry of the channel $W$ is not a major limitation: as long as we are interested only in the case when the input distribution $Q$ is uniform, the $E_{0}$ of a channel $W$ is the same as an associated symmetric channel $\tilde{W}$ as defined in Lemma 1.4 of [8]. Since the symmetrizing operator $W \rightarrow$ $\tilde{W}$ commutes with the polar transforms, all the conclusions of the paper are valid for arbitrary binary input channels as long as one evaluates all quantities under the uniform input distribution.

\section{ACKNOWLEDGMENT}

The author would like to thank Emre Telatar for helpful discussions.

\section{REFERENCES}

[1] A. Guillen i Fabregas, I. Land, and A. Martinez. Extremes of random coding error exponents. In Information Theory Proceedings (ISIT), 2011 IEEE International Symposium on, pages 2896 -2898, 31 2011-aug. 5 2011.

[2] R. G. Gallager. Information Theory and Reliable Communication. John Wiley \& Sons, Inc., New York, NY, USA, 1968.

[3] E. Arıkan. Channel polarization: a method for constructing capacityachieving codes for symmetric binary-input memoryless channels. IEEE Trans. Inf. Theor., 55(7):3051-3073, 2009.

[4] E. Arrkan and E. Telatar. BEC and BSC are $E_{0}$ extremal. Unpublished manuscript. July 2008.

[5] S. Arimoto. Information measures and capacity of order $\alpha$ for discrete memoryless channels. In Topics in information theory, I.Csiszar and P. Elias, editors, Amsterdam, The Netherlands, 1977. North-Holland Publishing Co.

[6] E. Telatar, Private Communications

[7] I. Sutskover, S. Shamai, and J. Ziv. Extremes of information combining. Information Theory, IEEE Transactions on, 51(4):1313 - 1325, April 2005.

[8] S.B. Korada, "Polar codes for channel and source coding," PhD Thesis, EPFL, Switzerland, 2009.

[9] M. Alsan. Channel polarization and polar codes. Technical Report. EPFL, Switzerland, 2012. http://infoscience.epfl.ch/record/176515

[10] I. S. Gradshteyn and I. M. Ryzhik. Table of Integrals, Series and Products. Academic Press Inc, 1994. 


\section{APPENDICES}

\section{A. Appendix A: Proof of Theorem 1}

We start with a lemma proved in [4] that expresses the function $E_{0}(\rho, W)$ in a more suitable form for the proof.

Lemma 1: [4] Given a symmetric B-DMC $W$, and a fixed $\rho \in[0,1]$, there exist a random variable $Z$ taking values in the $[0,1]$ interval such that

$$
E_{0}(\rho, W)=-\log \mathbb{E}[g(\rho, Z)]
$$

where

$$
g(\rho, z)=\left(\frac{1}{2}(1+z)^{\frac{1}{1+\rho}}+\frac{1}{2}(1-z)^{\frac{1}{1+\rho}}\right)^{1+\rho} .
$$

Moreover, the random variable $Z_{\mathrm{BEC}}$ of a binary erasure channel is $\{0,1\}$ valued. The random variable $Z_{\mathrm{BSC}}$ of a binary symmetric channel is a constant $z_{\mathrm{BSC}}$.

The proof of the lemma can be found in Lemma 4.1 [9].

Based on this representation, the function $R(\rho, W)$ equals

$$
R(\rho, W)=\frac{\mathbb{E}[-\partial g(\rho, Z) / \partial \rho]}{\mathbb{E}[g(\rho, Z)]} .
$$

To prove the theorem we need to introduce a set of lemmas, and corollaries to them.

Lemma 2: For a fixed value of $\rho$, the function $g(\rho, z)$ defined in Equation (16) is a concave decreasing function in the variable $z$.

The proof of the lemma is carried in Appendix A of [9].

Lemma 3: For fixed values of $\rho_{1}, \rho_{2} \in[0,1]$ such that $\rho_{1} \leq$ $\rho_{2}$, the function $\tilde{f}_{\rho_{1}, \rho_{2}}(t)$ defined as

$$
\tilde{f}_{\rho_{1}, \rho_{2}}(t)=\frac{\partial}{\partial \rho_{2}} g\left(\rho_{2}, g^{-1}\left(\rho_{1}, t\right)\right)
$$

is a concave function in the variable $t$.

The convexity analysis is tedious, and we refer the readers to Appendix D of [9] for a proof. We first prove Theorem 1 for the case $\rho_{1}=\rho_{2}$ in the next corollary.

Corollary 4: Under the same assumptions of Theorem 1, the following extremality property holds:

$$
R\left(\rho_{1}, W_{\mathrm{BSC}}\right) \leq R\left(\rho_{1}, W\right) \leq R\left(\rho_{1}, W_{\mathrm{BEC}}\right) .
$$

Proof: By the equality conditions in Equation (6), the denominator in Equation (17) is the same for all the channels. Then, the proof follows directly by the concavity of the function $\tilde{f}_{\rho_{1}}(t)$ in $t$, and the special structure of the $Z$ random variable corresponding to the channels $W_{\mathrm{BSC}}$, and $W_{\mathrm{BEC}}$. We define the random variable $T=g(\rho, Z)$. By the two sides of the Jensen's inequality for concave functions, i.e.

$$
\begin{aligned}
\tilde{f}_{\rho_{1}}(1)+\frac{\tilde{f}_{\rho_{1}}(1)-\tilde{f}_{\rho_{1}}\left(2^{-\rho_{1}}\right)}{1-2^{-\rho_{1}}}(\mathbb{E}[T]-1) \leq & \mathbb{E}\left[\tilde{f}_{\rho_{1}}(T)\right] \\
& \leq \tilde{f}_{\rho_{1}}(\mathbb{E}[T])
\end{aligned}
$$

Equation (18) follows ( $-\tilde{f}$ is convex).
Corollary 5: Under the same assumptions of Theorem 1, we have for $\rho_{2} \geq \rho_{1}$

$$
E_{0}\left(\rho_{2}, W_{\mathrm{BSC}}\right) \leq E_{0}\left(\rho_{2}, W\right) \leq E_{0}\left(\rho_{2}, W_{\mathrm{BEC}}\right) .
$$

Proof: By the continuity of $E_{0}(\rho, W)$ in the channel, it suffices to show that

$$
E_{0}\left(\rho_{1}, W_{\mathrm{BSC}}\right)<E_{0}\left(\rho_{1}, W\right)<E_{0}\left(\rho_{1}, W_{\mathrm{BEC}}\right)
$$

implies

$$
E_{0}\left(\rho_{2}, W_{\mathrm{BSC}}\right)<E_{0}\left(\rho_{2}, W\right)<E_{0}\left(\rho_{2}, W_{\mathrm{BEC}}\right) .
$$

From Theorem 1,

$$
E_{0}\left(\rho, W_{\mathrm{BSC}}\right)<E_{0}(\rho, W)<E_{0}\left(\rho, W_{\mathrm{BEC}}\right)
$$

implies

$$
R\left(\rho, W_{\mathrm{BSC}}\right)<R(\rho, W)<R\left(\rho, W_{\mathrm{BEC}}\right) .
$$

We define $F(\rho)=E_{0}(\rho, W)-E_{0}\left(\rho, W_{\mathrm{BEC}}\right)$, or $F(\rho)=$ $E_{0}\left(\rho, W_{\mathrm{BSC}}\right)-E_{0}(\rho, W)$. Noting that $R(\rho)=\frac{\partial}{\partial \rho} E_{0}(\rho)$, the corollary is implied by the following statement:

$$
F\left(\rho_{1}\right)<0 \text { and }\left(F(\rho)<0 \Rightarrow F^{\prime}(\rho)<0\right) \Rightarrow F\left(\rho_{2}\right)<0 .
$$

But this is true by elementary considerations on differential equations.

Now, we proceed with the proof of the theorem for the case $\rho_{2}>\rho_{1}$. By Lemma 3, the function $\tilde{f}_{\rho_{1}, \rho_{2}}(t)$ is concave in $t$. So, we can apply the two sides of Jensen's inequality to obtain

$$
\begin{aligned}
\mathbb{E}\left[-\tilde{f}_{\rho_{1}, \rho_{2}}\left(g\left(\rho_{1}, Z_{\mathrm{BSC}}\right)\right)\right] & \leq \mathbb{E}\left[-\tilde{f}_{\rho_{1}, \rho_{2}}\left(g\left(\rho_{1}, Z\right)\right)\right] \\
& \leq \mathbb{E}\left[-\tilde{f}_{\rho_{1}, \rho_{2}}\left(g\left(\rho_{1}, Z_{\mathrm{BEC}}\right)\right)\right] .
\end{aligned}
$$

On the other hand, as $E_{0}(\rho, W)=-\log \mathbb{E}[g(\rho, Z)]$ by Lemma 1, we know by Corollary 5 that

$$
\mathbb{E}\left[g\left(\rho_{2}, Z_{\mathrm{BEC}}\right)\right] \leq \mathbb{E}\left[g\left(\rho_{2}, Z\right)\right] \leq \mathbb{E}\left[g\left(\rho_{2}, Z_{\mathrm{BSC}}\right)\right] .
$$

Taking the ratios of the last two set of inequalities proves the extremality property stated in Equation (7).

\section{B. Appendix B: Proof of Theorem 2}

As we did in Appendix A, we start with two lemmas to express the functions $E_{0}\left(\rho, W^{+}\right)$, and $E_{0}\left(\rho, W^{-}\right)$in a similar form as in Lemma 1.

Lemma 4: Given a B-DMC $W$ and a fixed $\rho \in[0,1]$, there exist i.i.d. random variables $Z_{1}$ and $Z_{2}$ taking values in the $[0,1]$ interval such that

$$
E_{0}(\rho, W)=-\log \mathbb{E}[g(\rho, Z)]
$$

and

$$
E_{0}\left(\rho, W^{-}\right)=-\log \mathbb{E}\left[g\left(\rho, Z_{1} Z_{2}\right)\right]
$$

where $g(\rho, z)$ is given by (16). 
Lemma 5: Given a B-DMC $W$ and a fixed $\rho \in[0,1]$, there exist i.i.d. random variables $Z_{1}$ and $Z_{2}$ taking values in the $[0,1]$ interval such that

$$
E_{0}(\rho, W)=-\log \mathbb{E}[g(\rho, Z)]
$$

and

$$
\begin{aligned}
E_{0}\left(\rho, W^{+}\right)=-\log \mathbb{E} & {\left[\frac{1}{2}\left(1+Z_{1} Z_{2}\right) g\left(\rho, \frac{Z_{1}+Z_{2}}{1+Z_{1} Z_{2}}\right)\right.} \\
+ & \left.\frac{1}{2}\left(1-Z_{1} Z_{2}\right) g\left(\rho, \frac{Z_{1}-Z_{2}}{1-Z_{1} Z_{2}}\right)\right]
\end{aligned}
$$

where $g(\rho, z)$ is given by (16).

The proofs can be found in Lemmas 4.2, 4.3 in [9].

The proof of the extremality property for the $W^{-}$transformation, given in Equation (13), relies on the convexity of the function defined in the next lemma.

Lemma 6: We define the function $F_{z, \rho}(t):\left[2^{-\rho}, 1\right] \rightarrow$ $[g(\rho, z), 1]$ as

$$
F_{z, \rho}(t)=g\left(\rho, z g^{-1}(\rho, t)\right)
$$

where both $z, \rho \in[0,1]$. For fixed values of $\rho$ and $z$, the function $F_{z, \rho}(t)$ is convex with respect to the variable $t$.

The convexity analysis of this function is tedious. We refer the readers to Appendix B of [9] for a proof. By Lemmas 1 and 4 , we have

$$
\begin{aligned}
& \exp \left\{-E_{0}(\rho, W)\right\}=\mathbb{E}[g(\rho, Z)] \\
& \exp \left\{-E_{0}\left(\rho, W^{-}\right)\right\}=\mathbb{E}\left[g\left(\rho, Z_{1} Z_{2}\right)\right]
\end{aligned}
$$

where $Z_{1}$ and $Z_{2}$ are independent random variables. Moreover by Lemma 1 , we know $Z_{\mathrm{BSC}}=z_{\mathrm{BSC}}$ and $Z_{\mathrm{BEC}} \in\{0,1\}$. Hence,

$$
\exp \left\{-E_{0}\left(\rho, W_{\mathrm{BSC}}^{-}\right)\right\}=g\left(\rho, z_{\mathrm{BSC}} z_{\mathrm{BSC}}\right) .
$$

Let $\epsilon$ be the erasure probability of $W_{\mathrm{BEC}}$. Then, we have $P\left(Z_{\mathrm{BEC}}=0\right)=\epsilon$, and

$$
\exp \left\{-E_{0}\left(\rho, W_{\mathrm{BEC}}\right)\right\}=P\left(Z_{\mathrm{BEC}}=0\right)\left(1-2^{-\rho}\right)+2^{-\rho} .
$$

Since, it is known that the channel $W^{-}$is a BEC with erasure probability $2 \epsilon-\epsilon^{2}$, we get

$$
\begin{aligned}
& \exp \left\{-E_{0}\left(\rho, W_{\mathrm{BEC}}^{-}\right)\right\} \\
& \quad=\left[2 P\left(Z_{\mathrm{BEC}}=0\right)-P\left(Z_{\mathrm{BEC}}=0\right)^{2}\right]\left(1-2^{-\rho}\right)+2^{-\rho} .
\end{aligned}
$$

Moreover, given $E_{0}(\rho, W)=E_{0}\left(\rho, W_{\mathrm{BSC}}\right)$, we also have

$$
\mathbb{E}[g(\rho, Z)]=g\left(\rho, z_{\mathrm{BSC}}\right) .
$$

Therefore, using Jensen's inequality we obtain

$$
\begin{aligned}
\exp \left\{-E_{0}\left(\rho, W^{-}\right)\right\} & =\mathbb{E}_{Z_{1}}\left[\mathbb{E}_{Z_{2}}\left[F_{z_{1}, \rho}\left(g\left(\rho, Z_{2}\right)\right) \mid Z_{1}=z_{1}\right]\right] \\
& \geq \mathbb{E}_{Z_{1}}\left[F_{Z_{1}, \rho}\left(\mathbb{E}_{Z_{2}}\left[g\left(\rho, Z_{2}\right)\right]\right)\right] \\
& =\mathbb{E}_{Z_{1}}\left[F_{Z_{1}, \rho}\left(g\left(\rho, z_{\mathrm{BSC}}\right)\right]\right] \\
& \stackrel{(1)}{=} \mathbb{E}_{Z_{1}}\left[F_{Z_{\mathrm{BSC}}, \rho}\left(g\left(\rho, Z_{1}\right)\right)\right] \\
& \geq F_{z_{\mathrm{BSC}}, \rho}\left(\mathbb{E}_{Z_{1}}\left[g\left(\rho, Z_{1}\right)\right]\right) \\
& =\exp \left\{-E_{0}\left(\rho, W_{\mathrm{BSC}}^{-}\right)\right\}
\end{aligned}
$$

where (1) follows by symmetry of the variables $Z_{1}$ and $z_{\mathrm{BSC}}$. Similarly, given $E_{0}(\rho, W)=E_{0}\left(\rho, W_{\mathrm{BEC}}\right)$, we have

$\mathbb{E}[g(\rho, Z)]=\mathbb{E}\left[g\left(\rho, Z_{\mathrm{BEC}}\right)\right]=P\left(Z_{\mathrm{BEC}}=0\right)\left(1-2^{-\rho}\right)+2^{-\rho}$.

Due to convexity, we also know the following inequality holds:

$$
F_{z, \rho}(t) \leq 1+\frac{g(\rho, z)-1}{2^{-\rho}-1}(t-1) .
$$

Therefore,

$$
\begin{aligned}
& \exp \left\{-E_{0}\left(\rho, W^{-}\right)\right\} \\
= & \mathbb{E}_{Z_{1}}\left[\mathbb{E}_{Z_{2}}\left[F_{Z_{1}, \rho}\left(g\left(\rho, Z_{2}\right)\right) \mid Z_{1}=z_{1}\right]\right] \\
\leq & \mathbb{E}_{Z_{1}}\left[1+\frac{g\left(\rho, Z_{1}\right)-1}{2^{-\rho}-1}\left(\mathbb{E}_{Z_{2}}\left[g\left(\rho, Z_{2}\right)\right]-1\right)\right] \\
= & 1+\frac{\mathbb{E}_{Z_{1}}\left[g\left(\rho, Z_{1}\right)\right]-1}{2^{-\rho}-1}\left(\mathbb{E}_{Z_{2}}\left[g\left(\rho, Z_{2}\right)\right]-1\right) \\
= & \frac{2^{-\rho}-1+\left[P\left(Z_{\mathrm{BEC}}=0\right)\left(1-2^{-\rho}\right)+2^{-\rho}-1\right]^{2}}{2^{-\rho}-1} \\
= & {\left[2 P\left(Z_{\mathrm{BEC}}=0\right)-P\left(Z_{\mathrm{BEC}}=0\right)^{2}\right]\left(1-2^{-\rho}\right)+2^{-\rho} } \\
= & \exp \left\{-E_{0}\left(\rho, W_{\mathrm{BEC}}^{-}\right)\right\} .
\end{aligned}
$$

This concludes the proof for the $W^{-}$transformation.

Now, we sketch the proof of the extremality property for the $W^{+}$transformation, given in Equation (14).

We define the function $h_{\rho}\left(z_{1}, z_{2}\right)$ as

$$
\begin{aligned}
& h_{\rho}\left(z_{1}, z_{2}\right)=\frac{1}{2}\left(1+z_{1} z_{2}\right) g\left(\rho, \frac{z_{1}+z_{2}}{1+z_{1} z_{2}}\right) \\
&+\frac{1}{2}\left(1-z_{1} z_{2}\right) g\left(\rho, \frac{z_{1}-z_{2}}{1-z_{1} z_{2}}\right)
\end{aligned}
$$

where $\rho, z_{1}, z_{2} \in[0,1]$. Note that $h_{\rho}\left(z_{1}, z_{2}\right)$ is symmetric in the variables $z_{1}$, and $z_{2}$.

Lemma 7: Let the function $H_{z, \rho}(t):\left[2^{-\rho}, 1\right] \rightarrow$ $\left[2^{-\rho}, g(\rho, z)\right]$ be defined as

$$
H_{z, \rho}(t)=h_{\rho}\left(g^{-1}(\rho, t), z\right)
$$

where both $z, \rho \in[0,1]$. For fixed values of $\rho$ and $z$, the function $H_{z, \rho}(t)$ is concave with respect to the variable $t$.

We refer the readers to Appendix F of [9] for a proof.

The proof of the theorem for the $W^{+}$transformation can be completed following similar steps to the $W^{-}$case. By Lemma 5 , we have $\mathbb{E}\left[h_{\rho}\left(Z_{1}, Z_{2}\right)\right]=\exp \left\{-E_{0}\left(\rho, W^{+}\right)\right\}$. We define $T_{1}=g\left(\rho, Z_{1}\right)$, and $T_{2}=g\left(\rho, Z_{2}\right)$. Then, using the concavity of the function $H_{z, \rho}(t)$ with respect to $t$, and symmetry of $Z_{1}$ and $Z_{2}$, we obtain

$$
\begin{aligned}
& \exp \left\{-E_{0}\left(\rho, W^{+}\right)\right\}=\mathbb{E}\left[H_{g^{-1}}\left(\rho, T_{2}\right), \rho\left(T_{1}\right)\right] \\
& \leq h_{\rho}\left(z_{\mathrm{BSC}}, z_{\mathrm{BSC}}\right)=\exp \left\{-E_{0}\left(\rho, W_{\mathrm{BSC}}^{+}\right)\right\}
\end{aligned}
$$

and

$$
\begin{aligned}
& \exp \left\{-E_{0}\left(\rho, W^{+}\right)\right\}=\mathbb{E}\left[H_{g^{-1}\left(\rho, T_{2}\right), \rho}\left(T_{1}\right)\right] \\
\geq & 2^{-\rho}+P\left(Z_{\mathrm{BEC}}=0\right)^{2}\left(1-2^{-\rho}\right)=\exp \left\{-E_{0}\left(\rho, W_{\mathrm{BEC}}^{+}\right)\right\} .
\end{aligned}
$$

